\title{
Synergistic antifungal indolecarbazoles from Streptomyces sp. CNS-42 associated with traditional Chinese medicine Alisma orientale
}

\author{
Mei Liu ${ }^{1,2,8}$, Pei Huang ${ }^{1,2,8}$, Qian Wang ${ }^{1,2}$, Biao Ren ${ }^{3}$, Ayokunmi Oyeleye ${ }^{4}$, Miaomiao Liu ${ }^{1,2}$, Jingyu Zhang ${ }^{1,5}$, \\ Xiaolin $\mathrm{Li}^{6,7}$, Xiaoping Zhang ${ }^{6}$, Lixin Zhang ${ }^{1,5}$ and Xueting Liu ${ }^{1,5}$
}

The Journal of Antibiotics (2017) 70, 715-717; doi:10.1038/ja.2016.160; published online 11 January 2017

Multi-drug resistance of pathogenic microorganisms is a serious threat to human health. In particular, Candida albicans, the most common opportunistic clinical fungal pathogen is one of the major causes of systemic infections, resulting in an estimated $30 \%$ of severe fungal infections, with mortality rate reaching nearly $40 \%{ }^{1,2}$ Widespread and repeated use of current drugs, particularly azoles, have contributed to the rapid occurrence of antifungal drug resistance, ${ }^{3,4}$ and most screening approaches for new drugs which target essential genes and fungal pathogens are likely to generate resistance over time. Given the high mortality rate resulting from fungal infections in immunocompromised patients and the limited number of highly effective, yet safe treatment agents, the development of new antifungal therapeutics is critical. ${ }^{1,2,5}$ A strategy limiting the pressure on drug targets would increase the lifespan of antifungal agents and reduce the frequency of treatment failures. ${ }^{3,6}$ For instance, using synergistic drugs aimed at more than one target and slowing down the emergence of drug-resistant pathogens will be one of the key approaches to antifungal therapy. Our research group demonstrated that beauvericin (BEA) showed strong synergism with ketoconazole against diverse fungal pathogens both in vitro and in vivo, ${ }^{5}$ and that this synergetic effect was not caused by their pharmacokinetic interaction. ${ }^{7}$

Looking for natural products with potentially new Mode of Action from untapped sources provides an essential strategy. Endophytic microbes associated with traditional Chinese medicines provide a variety of active compounds for therapeutics. In addition, one of the major limiting factors in natural products drug discovery industry is that pharmaceutical companies have been traditionally designed to target individual factors in a disease system, but diseases are complex in nature and vulnerable at multiple attacks. Therefore, a systematic novel synergistic drug screening approach based on a multifactorial principle is urgently needed, especially from our Microbial Natural Product Libraries (MNPL).

A high-throughput synergy screening (HTSS) method has been developed in our lab for identifying synergistic microbial natural products in combination with a low dosage of ketoconazole against fungal pathogens in vitro. We used a much-reduced dosage compared with the established therapeutic dosage of KTC to sensitize and potentiate fungal pathogens while screening for potent co-drugs in an established microbial natural product library. This procedure takes advantage of KTC at low dosage concentration and screens for those synergistic partners from broad microbial natural products to enhance efficacy.

A screening on in-house MNPL has led to the discovery of active beauvericin and avermectins (Supplementary Table S2). ${ }^{5,8,9}$ Combinations of BEA $\left(0.5 \mathrm{mg} \mathrm{kg}^{-1}\right)$ and KTC $\left(0.5 \mathrm{mg} \mathrm{kg}^{-1}\right)$ prolonged survival of the host infected with Candida parapsilosis and reduced fungal colony counts in animal organs including kidneys, lungs and brains in an immunocompromised mouse model..$^{5}$ Further screening on a sub-library of microbial natural products has been done, contained 900 crude extracts from the fermentation broth of endophytic bacteria 300 strains on three different media (AM2, NM2 and MPG). HTSS on these extracts gave a $1.2 \%$ hit rate and generated $90 \%$ of the maximum activity in combination with $1 / 4$ MIC of KTC. In addition, the microbial extract alone had little to no effect. Among these hits, along with the chemo-type analysis, an extract derived from the fermentation broth of endophytic actinomycete Streptomyces sp. (CNS-42) was highlighted with MIC values of 6.25 and $3.125 \mu \mathrm{g} \mathrm{ml}^{-1}$ against C. albicans (SC 5314) and C. albicans (SC 5314) synergistic model, respectively. Bioassay-directed fractionation

\footnotetext{
${ }^{1}$ Chinese Academy of Sciences Key Laboratory of Pathogenic Microbiology and Immunology, Institute of Microbiology, Chinese Academy of Sciences, Beijing, China; ${ }^{2}$ University of Chinese Academy of Sciences, Beijing, China; ${ }^{3}$ State Key Laboratory of Oral Diseases, West China Hospital of Stomatology, Sichuan University, Chengdu, China; ${ }^{4}$ Universiti Putra Malaysia, Faculty of Biotechnology and Biomolecular Sciences, Kuala Lumpur, Malaysia; ${ }^{5}$ State Key Laboratory of Bioreactor Engineering, East China University of Science \& Technology, Shanghai, China; ${ }^{6}$ Department of Microbiology, College of Resource and Environment, Sichuan Agricultural University, Chengdu, China and ${ }^{7}$ Soil and Fertilizer Institute, Sichuan Academy of Agricultural Sciences, Chengdu, China

8These authors contributed equally to this work.

Correspondence: Professor L Zhang or Professor X Liu, Chinese Academy of Sciences Key Laboratory of Pathogenic Microbiology and Immunology, Institute of Microbiology, Chinese Academy of Sciences, No.1 Beichen West Road, Chaoyang District, Beijing 100101, China.

E-mail: Izhang03@gmail.com or liuxt2010@gmail.com

Received 3 November 2016; revised 20 November 2016; accepted 29 November 2016; published online 11 January 2017
} 


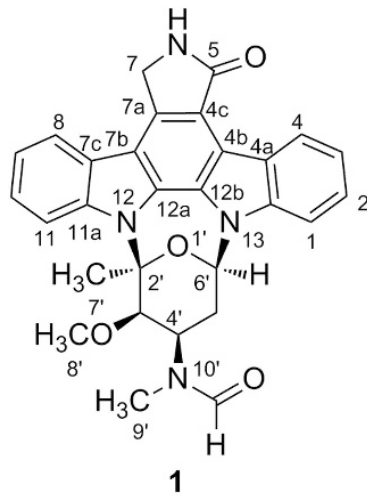

1
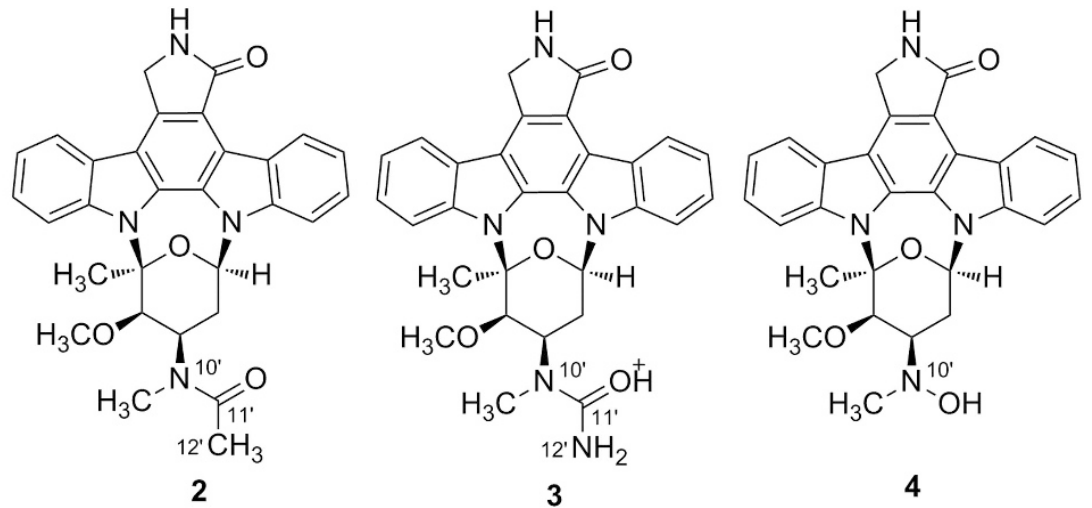

Figure 1 Structures of compounds 1-4.

Table 1 Antimicrobial activities of compounds 1-4

$M I C\left(\mu g l^{-1}\right)$

\begin{tabular}{|c|c|c|c|c|c|c|}
\hline Organism (strain) & Staurosporine & 1 & 2 & 3 & 4 & Control \\
\hline BCG (Mycobacterium bovis Pasteur 1173P2) & $>100$ & $>100$ & $>100$ & $>100$ & $>100$ & $0.05^{a}$ \\
\hline Methicillin-resistant S. aureus ${ }^{b}$ & $>100$ & $>100$ & $>100$ & $>100$ & $>100$ & $1^{\mathrm{c}}$ \\
\hline B. subtilis (ATCC 6633) & 12.5 & $>100$ & $>100$ & $>100$ & $>100$ & $0.5^{c}$ \\
\hline C. albicans (SC 5314) & 0.1 & $>100$ & 100 & $>100$ & $>100$ & $0.016^{\mathrm{e}}$ \\
\hline C. albicans (SC 5314) synergistic ${ }^{f}$ & 0.1 & 6.25 & 0.78 & $>100$ & $>100$ & $1 \mathrm{~g}$ \\
\hline
\end{tabular}

Abbreviations: BCG, Bacillus Calmette-Guérin, MRSA, methicillin-resistant S. aureus.

alsoniazid.

'DRSA, Clinical isolates, Beijing Chao-yang Hospital, Beijing, China.

'Vancomycin.

${ }^{\mathrm{d}}$ Ciprofloxacin.

eKetoconazole.

'The synergistic activity of tested compounds with triazole against $\mathrm{C}$. albicans.

gCyclosporin A.

of a large-scale ( $42 \mathrm{~L})$ culture of CNS-42 yielded four known indolecarbazoles (1-4) (Figure 1). 1 and 2 showed potent synergistic antifungal activities with MIC values of 6.25 and $0.78 \mu \mathrm{g} \mathrm{ml}^{-1}$, respectively, when combined with the addition of $25 \%$ of the MIC of ketoconazole in the fungal suspension, but weak or no antifungal activity $\left(\mathrm{MIC} \geqslant 100 \mu \mathrm{g} \mathrm{ml}^{-1}\right.$ ) when tested singly. This paper describes the finding of new activity of indolecarbazoles for further pharmaceutical application study.

The structures of staurosporines (1-4) (Figure 1) were elucidated based on combined comparison of their UV, high resolution electrospray ionization mass spectroscopy (HRESIMS) analysis and ${ }^{1} \mathrm{H}$ NMR with those of reported literatures. ${ }^{10,11}$ Staurosporines (1-4) showed typical UV-visible spectra similar to those of known indolecarbazoles (supporting information). 1 was obtained as yellowish powder and its molecular formula $\mathrm{C}_{29} \mathrm{H}_{26} \mathrm{~N}_{4} \mathrm{O}_{4}$ was deduced from its HRESIMS ([M $+\mathrm{H}]^{+}, m / z$ 495.2089, cald. 495.2027) (Supplementary Figure S2). The UV spectrum of 1 which was very similar to that of staurosporine revealed that 1 contained a skeleton of indole carbazole (Supplementary Figure S1). The ${ }^{1} \mathrm{H}$ NMR spectrum (Supplementary Table S1 and Supplementary Figure S3) of 1 was almost identical to that of staurosporine, ${ }^{11}$ except for the presence of an $\mathrm{N}$-formyl group $\left(\delta_{\mathrm{H}}\right.$ 9.28). This revealed the $\mathrm{NH}$ group on staurosporine was replaced by an $\mathrm{N}-\mathrm{CHO}$ group on $\mathbf{1}$. The structure of $\mathbf{1}$ was determined to be $\mathrm{N}$-formyl-staurosporine by comparison of its ${ }^{1} \mathrm{H}$ NMR data with those reported previously. ${ }^{12}$
Staurosporins 2-4 were all obtained as yellowish powder. Their molecular formulae deduced from their HRESIMS are $\mathrm{C}_{30} \mathrm{H}_{28} \mathrm{~N}_{4} \mathrm{O}_{4}$ $\left([\mathrm{M}+\mathrm{H}]^{+}, m / z\right.$ 509.2253, cald. 509.2183) (Supplementary Figure S4), $\mathrm{C}_{29} \mathrm{H}_{27} \mathrm{~N}_{5} \mathrm{O}_{4}\left([\mathrm{M}+\mathrm{H}]^{+}, m / z 510.2210\right.$, cald. 510.2136) (Supplementary Figure S6), $\mathrm{C}_{28} \mathrm{H}_{26} \mathrm{~N}_{4} \mathrm{O}_{4}\left([\mathrm{M}+\mathrm{H}]^{+}, m / z\right.$ 483.2093, cald. 483.2027; Supplementary Figure S8) for 2, 3 and 4, respectively. The structures of $\mathbf{2 - 4}$ as determined by their UV and ${ }^{1} \mathrm{H}$ NMR (Supplementary Figures S5, respectively) (Supplementary Table S1) spectra were very similar to those of 1 except for the replacement of the proton of the $\mathrm{N}$-formyl group on 1 by a methyl group $\left(\delta_{\mathrm{H}} 2.08\right.$, s) in 2 , an amine group $\left(\delta_{\mathrm{H}} 6.03, \mathrm{~s}\right)$ in $\mathbf{3}$ and an extra hydroxyl group $\left(\delta_{\mathrm{H}} 7.78, \mathrm{~s}\right)$ in $\mathbf{4 .} 2$ was deduced as $\mathrm{N}$-acetyl-staurosporin, ${ }^{13} 3$ as $\mathrm{N}$-carboxamidostaurosporine $e^{14}$ and 4 as $4^{\prime}-\mathrm{N}$-hydroxy-staurosporine $\mathrm{e}^{15}$ by comparing ${ }^{1} \mathrm{H}$ NMR data with those of previous reports.

The antimicrobial activities of $\mathbf{1} \mathbf{- 4}$ have been evaluated (Table 1). All compounds did not show activities against Bacillus CalmetteGuérin, Staphylococcus aureus, Bacillus subtilis, Pseudomonas aeruginosa (Table 1). Interestingly, both 1 and 2 showed strong synergistic antifungal activities with MIC values of 6.25 and $0.78 \mu \mathrm{g} \mathrm{ml}^{-1}$ with the addition of $25 \%$ of the MIC of ketoconazole in the fungal suspension ( fractional inhibitory concentration index $(\mathrm{FICI})<0.31$ and $=0.26$, respectively).

The indolocarbazole (ICZ) family of natural products is a source of lead compounds with potential therapeutic applications in the treatment of cancer and other diseases. Since the isolation of the first ICZ in 1977, that is, staurosporine, which was renamed and 
structurally corrected afterward, ${ }^{15,16}$ this family of compounds has attracted the attention of many researchers due to their variety of chemical structures and biological activities. There are more than 150 compounds identified from various natural sources including slime molds, marine invertebrates, actinomycetes, cyanobacteria and others. ${ }^{17}$ ICZs display a variety of biological activities, including anticancer, antibacterial, antifungal and antiviral activities. ${ }^{18-20}$

The ICZ derivatives are capable of targeting protein kinases and DNA topoisomerases as required for the treatment of a range of important human diseases, hence the quest for more active and novel ICZ analogs and/or new activities.

In a previous study, we reported the antifungal and plant growth promoting activities of staurosporine from endophytic actinobacteria. ${ }^{10}$ In our lab, the well-developed HTSS platform has highlighted two significant products, beauvericin and avermectin (Supplementary Table S2), which showed outstanding synergistic activities in combination with a low dosage of KTC against fungal pathogens in vitro. In this study, we report two more molecules 1 and 2 identified with good synergistic antifungal activity. However, no significant activity was observed on fungal pathogens when applied singly. This indicates a potential new Mode of Action of these molecules against fungal pathogens. This report might provide a potential pharmaceutical application of the discovery of antifungal drug leads, especially for the treatment of diseases caused by ketoconazole resistant pathogens.

\section{DEDICATION}

This article is dedicated to Dr Satoshi Ōmura on his Nobel Prize with two other prominent scientists in Physiology or Medicine 2015 for the discoveries concerning a novel therapy against infections caused by roundworm parasites. As a leading advocator and mentor in the field of Microbial Natural Product, he discovered the 'wonder anthelmintic' drug, avermectin (AVM) from Streptomyces avermitilis. Giving hope to patients suffering from 'river blindness' caused by Onchocerca volvulus. Additional activities include evidence that AVM family selectively induces mortality in Mycobacterium tuberculosis including some multi-drug resistant clinical isolates. We identified another analog of AVM re-sensitizes clinical methicillin-resistant $S$. aureus (MRSA) to methicillin and can also synergistically sensitize fungal pathogens, such as Candida albicans to azole drugs. Since wild-type strains can only produce low-level of AVM, the titer improvement becomes very important for the fermentation industry. Under our great team efforts, the production of avermectins had increased from $9 \mu \mathrm{g} \mathrm{ml}^{-1}$ to $9000 \mu \mathrm{g} \mathrm{ml}^{-1}$ while the cost reduced more than 50 fold and it is exclusively made in China. We are also greatly inspired by other contributions from Dr Ōmura in natural product based drug discovery.

\section{CONFLICT OF INTEREST}

The authors declare no conflict of interest.

\section{ACKNOWLEDGEMENTS}

This work was supported in part by the National Program on Key Basic Research Project (973 program, 2013CB734000), by grants from the China Ocean Mineral Resources R\&D Association (DY125-15-T-07), the National Natural Science Foundation of China (81573341, 31670052, 31170095, $81102369,31430002,81302678,31320103911)$, the Ministry of Science and Technology of China (2013ZX10005004-005 and 2011ZX09102-011-11) and the European Union's Seventh Framework Programme (FP7/2007-2013) under grant agreement no. 312184 .

1 Patterson, T. F. Advances and challenges in management of invasive mycoses. Lancet 366, 1013-1025 (2005)

2 Brown, G. D., Denning, D. W. \& Levitz, S. M. Tackling human fungal infections. Science 336, 647-647 (2012).

3 Anderson, J. B. Evolution of antifungal-drug resistance: mechanisms and pathogen fitness. Nat. Rev. Microbiol. 3, 547-556 (2005).

4 Cowen, L. E. The evolution of fungal drug resistance: modulating the trajectory from genotype to phenotype. Nat. Rev. Microbiol. 6, 187-198 (2008).

5 Zhang, L. et al. High-throughput synergy screening identifies microbial metabolites as combination agents for the treatment of fungal infections. Proc. Natl Acad. Sci. USA 104, 4606-4611 (2007).

6 Kohli, A., Smriti, Mukhopadhyay, K., Rattan, A. \& Prasad, R. In vitro low-level resistance to azoles in Candida albicans is associated with changes in membrane lipid fluidity and asymmetry. Antimicrob. Agents Chemother. 46, 1046-1052 (2002).

7 Mei, L., Zhang, L. \& Dai, R. An inhibition study of beauvericin on human and rat cytochrome P450 enzymes and its pharmacokinetics in rats. J. Enzyme. Inhib. Med. Chem. 24, 753-762 (2009).

8 Zhuo, Y. et al. Reverse biological engineering of hrdB to enhance the production of avermectins in an industrial strain of Streptomyces avermitilis. Proc. Natl Acad. Sci. USA 107, 11250-11254 (2010).

9 Guo, H. et al. Reversal of meticillin resistance in Staphylococcus aureus by the anthelmintic avermectin. Int. J. Antimicrob. Agents 44, 274-279 (2014).

$10 \mathrm{Li}, \mathrm{X}$. et al. Staurosporine from the endophytic Streptomyces sp. strain CNS-42 acts as a potential biocontrol agent and growth elicitor in cucumber. Antonie Van Leeuwenhoek 106, 515-525 (2014).

$11 \mathrm{Li}, \mathrm{X}$. B. et al. A new staurosporine analog from Actinomycetes Streptomyces sp. (172614). J. Asian Nat. Prod. Res. 13, 765-769 (2011).

12 Cai, Y., Fredenhagen, A., Hug, P. \& Peter, H. A nitro analogue of staurosporine and other minor metabolites produced by a Streptomyces longisporoflavus strain. J. Antibiot. (Tokyo) 48, 143-148 (1995).

13 Barrabee, E., Horan, A., Gentile, F. \& Patel, M. Indolocarbazoles from saccharothrix aerocolonigenes subsp. copiosa subsp. nov. scc 1951 ATCC 53856. WO/1991/009034 (1991).

$14 \mathrm{Wu}, \mathrm{S}$. et al. N-Carboxamido-staurosporine and selina-4(14), 7(11)-diene-8, 9-diol, new metabolites from a marine Streptomyces sp. J. Antibiot. (Tokyo) 59, 331-337 (2006).

15 Omura, S. et al. A new alkaloid AM-2282 of Streptomyces origin. Taxonomy, fermentation, isolation and preliminary characterization. J. Antibiot. (Tokyo) 30, 275-282 (1977).

16 Furusaki, A. et al. X-Ray crystal structure of staurosporine: a new alkaloid from a Streptomyces strain. J. Chem. Soc. Chem. Commun. 18, 800-801 (1978).

17 Sánchez, C., Méndez, C. \& Salas, J. A. Indolocarbazole natural products: occurrence, biosynthesis, and biological activity. Nat. Prod. Rep. 23 1007-1045 (2006).

18 Prudhomme, M. Rebeccamycin analogues as anti-cancer agents. Eur. J. Med. Chem. 38, 123-140 (2003).

19 Knölker, H. J. \& Reddy, K. R. Isolation and synthesis of biologically active carbazole alkaloids. Chem. Rev. 102, 4303-4427 (2002).

20 Long, B. H., Rose, W. C., Vyas, D. M., Matson, J. A. \& Forenza, S. Discovery of antitumor indolocarbazoles: rebeccamycin, NSC 655649, and fluoroindolocarbazoles. Curr. Med. Chem. Anticancer. Agents 2, 255-266 (2002).

Supplementary Information accompanies the paper on The Journal of Antibiotics website (http://www.nature.com/ja) 